\title{
REINGENIERÍA DE PROCESOS
}

\section{BUSINESS REINGENERING PROCESS}

Recepción: 23/05/2017

Aceptación: 13/08/2017

Publicación: 22/12/2017

Gerard Pérez Andrés ${ }^{1}$

Victor Gisbert Soler ${ }^{2}$

Elena Pérez Bernabeu ${ }^{3}$

1. Ingeniero industrial especialidad eléctrica. Máster Universitario en Organización y Logística. Universidad Politécnica de Valencia - Campus de Alcoy. (España). E-mail: geprean@alumni.upv.es

2. Doctor Ingeniero Industrial. Departamento de Estadística e Investigación Operativa Aplicadas y Calidad. Universidad Politécnica de Valencia. (España). E-mail: vgisber@eio.upv.es

3. Doctor Ingeniero en Organización Industrial. Departamento de Estadística e Investigación Operativa Aplicadas y Calidad. Universidad Politécnica de Valencia. (España). E-mail: elpeber@eio.upv.es

Citación sugerida:

Pérez Andrés, G., Gisbert Soler, V. y Pérez Bernabeu, E. (2017). Reingeniería de procesos. 3C Empresa: investigación y pensamiento crítico, Edición Especial, 81-91. DOI: $<$ http://dx.doi.org/10.17993/3cemp.2017.especial.81-91/>. 


\section{RESUMEN}

La reingeniería de procesos puede suponer una gran ventaja competitiva para las empresas, dado que si se realiza de forma adecuada, rediseñando los procesos para obtener mejoras, aumentando así el rendimiento e incluso reduciendo costes.

En este artículo se estudiará la metodología utilizada para la reingeniería de procesos, partiendo desde cómo surgió el proceso de reingeniería para mejora del rendimiento en organizaciones a partir de pequeños cambios tanto en sistemas productivos como organizacionales. Hay que destacar la importancia de la implicación de todas las partes de la organización para poder cumplir los objetivos definidos por la misma.

\section{ABSTRACT}

Process reengineering can be a great competitive advantage for companies, since if done properly, redesigning processes to obtain improvements, thus increasing performance and even reducing costs.

In this article we will study the methodology used for process reengineering, starting from the emergence of the process of reengineering to improve performance in organizations based on small changes in both production and organizational systems. It is necessary to emphasize the importance of the implication of all the parts of the organization in order to be able to fulfill the objectives defined by the same.

\section{PALABRAS CLAVE}

Reingeniería de procesos, rediseño de la organización, innovación.

\section{KEY WORDS}

Business Rengineering process, Organization redesign, innovation. 


\section{INTRODUCCIÓN}

La reingeniería de procesos es una herramienta administrativa la cual consiste en estudiar los procesos productivos de organizaciones de cualquier sector, y a través del cual se pueden rediseñar procesos productivos realizando modificaciones en dichos procesos, los cuales van a repercutir en el rendimiento medio de costes, tiempo de ciclo, calidad del servicio y calidad del producto.

Para llevar a cabo la reingeniería de procesos las personas que formen parte de la organización deben tener claras las metas que quiere alcanzar la organización, la forma de alcanzar dichas metas y que indicadores van a medir si se han alcanzado dichas metas.

Además de conocer las metas u objetivos a alcanzar, las personas que componen la organización deben de implicarse completamente en la implantación de las mejoras propuestas para rediseñar los procesos productivos, dado que esto será primer paso para que las mejoras implantadas sean efectivas.

\section{ANTECEDENTES}

- ANTECEDENTE 1: Reingeniería de procesos: conceptos, enfoques y nuevas aplicaciones.

$>$ Introducción a la reingeniería de procesos

$>$ Efectos y usos de la reingeniería de procesos.

$>$ La reingeniería en el sector de la información. Aplicaciones prácticas.

$>$ Gestión por procesos y reingeniería

- ANTECEDENTE 2: La reingeniería de procesos: una herramienta gerencial para la innovación y mejora de la calidad en las organizaciones (Rodrigo Ospina Duque)

Se caracteriza por:

D Estudio del impacto de las tendencias económicas, tecnológicas y enfatiza en las fuerzas del cambio.

$>$ Alternativas de las organizaciones para adaptarse a los cambios (reingeniería de procesos, la rápida reingeniería y la reingeniería de negocios).

$>$ Comportamientos fundamentales a tener en cuenta en procesos de reingeniería.

- ANTECEDENTE 3: Reingeniería de procesos y calidad total en la práctica empresarial: estudio de un caso (BERNABÉ ESCOBAR PÉREZ, JOSÉ MARÍA GONZÁLEZ GONZÁLEZ).

Se caracteriza por la comparativa entre la gestión de la calidad total y la reingeniería de procesos, se centra en la diferencia de los objetivos, factores básicos, implantación, repercusiones organizativas y compatibilidad entre ambas. 
Se diferencia con el ANTECEDENTE 1 en que aborda el tema de reingeniería de procesos centrándose en un caso práctico mientras que en el 1 lo hace de forma general.

- ANTECEDENTE 4: Reingeniería de procesos de negocio: Análisis y discusión de factores críticos a través de un estudio de caso (Bernabé Escobar Pérez, José María González González).

Se asemeja al antecedente 1 en estudiar los puntos críticos en la implantación de un BPR (comportamiento ante la implantación de un BPR).

Se centra en el método de investigación utilizado para conseguir el análisis de los factores críticos de un BPR.

> Resultados obtenidos del estudio en un caso práctico de una empresa que implanta un BPR.

\section{METODOLOGÍA}

La reingeniería en sus principios se define según Hammer como:

<<Reingeniería es el concepto actual que se le da a los cambios drásticos que sufre una organización al ser restructurados sus procesos.>>

Podemos diferenciar metodologías de procesos de reingeniería dependiendo del punto de vista del autor:

Según Lefcovich: $<$ La reingeniería debería ser un método para aprovechar las fortalezas internas de le empresa, y eliminar las debilidades de la misma , aprovechado oportunidades externas >>

La metodología que plantea Lefcovich sería la siguiente:

1. Análisis de la situación.

2. Diagnóstico

3. Diseño de la nueva organización

4. Implementación del nuevo diseño de la organización.

En este caso, cabe mencionar que después de la implantación del nuevo diseño de la organización existe resistencia al cambio por parte del personal, el cual se debe a miedo a que las cosas no salgan con los resultados esperados por tanto faltaría añadir los siguientes pasos:

5. Validación del buen funcionamiento de las mejoras implantadas. 
6. En caso de existir deficiencias o falta de alguna mejora, se realizaría un rediseño de la organización hasta alcanzar los objetivos establecidos.

Según el estudio de Bernabé Escobar Pérez y J.M. González González, el BPR (Business process rengineering) está basado en la literatura existente sobre el tema de los autores Hammer y champy la cual define el BPR como: <<La revisión fundamental y el rediseño radical de procesos para alcanzar mejoras espectaculares en medidas críticas $y$ contemporáneas del rendimiento tales como costes, calidad, servicio y rapidez.>>

La Metodología utilizada para un caso práctico en la implantación de un BRP según dichos autores consiste en:

1. Inducción a la reingeniería: periodo en el cual la alta dirección debe persuadir a los integrantes de la organización de la necesidad de afrontar cambios importantes en la misma.

2. Identificación y análisis de oportunidades de rediseño: consiste en identificar que procesos de negocio necesitan ser rediseñados en función a los objetivos establecidos en el BPR, para ello hay que identificar todos los procesos que realiza la organización y clasificarlos según la urgencia que necesiten de ser rediseñados.

Existen criterios para priorizar en unos procesos sobre otros a la hora de rediseñarlos:

- Procesos que presentan problemas

- Importancia o impacto sobre clientes externos.

- Viabilidad de que se puedan realizar rediseño con éxito.

Las técnicas utilizadas para la recogida de información son:

a) Observación participante

b) Entrevistas semiestructuradas a todos los empleados de la empresa.

c) Observación no participante.

d) Análisis de documentos.

e) Conversaciones informales con los empleados

f) Análisis del sistema SI económico-financiero de la empresa.

3. Rediseño de los procesos:

Es la parte más creativa del BPR en la cual hay que innovar o rediseñar los procesos para que estos sustituyan a los procesos anteriores, consiguiendo así mayor eficiencia y eficacia que en los procesos anteriores.

Existen dos métodos para el rediseño del puesto: 
- Método hoja en blanco: defendido por los ortodoxos consiste en diseñar el proceso desde cero, puesto que para ellos reingeniería consiste en eliminar lo anterior y comenzar con lo nuevo. (hammer y champy)

- Otros defienden (hill y Collins) que dicha creación del proceso desde cero constituye una amenaza para la reputación de la empresa o confianza de los clientes en la misma, por lo que realizan un enfoque "hoja en sucio" que consiste en rediseñar el proceso a partir del proceso anterior, sin empezar desde cero.

4. Implantación de los procesos rediseñados:

Consiste en sustituir los procesos anteriores por los actuales, ya sean completamente nuevos o actualizaciones de los anteriores.

En esta etapa es posible que se muestre resistencia al cambio por los empleados, esto constituye una de las principales causas de fracaso de la implantación del BPR.

Cabe destacar que para que la implantación del BPR sea efectiva ha de existir compromiso de la alta dirección, liderazgo, comunicación, participación de los empleados y estructura de equipo.

En otro estudio realizado por los autores Bernabé Escobar Pérez y J.M. González González, utiliza la misma definición para BPR citando la frase de Hammer y Champy.

En este caso se relaciona el tema de calidad total con la reingeniería de procesos:

La TQM (Total Quality Managment) se define como « Una forma de mejorar continuamente el rendimiento a cada nivel de operación, en cada área fundacional de una organización, utilizando todos los recursos humanos y capital disponible»> (Brocka y Brocka 1992).

La TQM se centra en actividades de planificación, dirección, organización, control y aseguramiento de la calidad.

Los tres principios de TQM son:

- Orientación al cliente para satisfacer sus necesidades

- Mejora continua realizando revisión de procesos (reingeniería de procesos).

- Trabajo en equipo fomentando la colaboración de empleados, clientes y trabajadores.

Se pueden observar grandes semejanzas entre el BPR y el TQM, entre ellas cabe destacar, el enfoque a mejora de los procesos, orientado a los clientes, así como la necesidad de que exista gran implicación de Alta dirección, empleados, etc.

En el caso analizado en dicho estudio relacionando TQM y BPR se puede distinguir la siguiente metodología utilizada para la mejora de la calidad total utilizando reingeniería de procesos:

1) Definición de Procesos, Subprocesos, clientes, etc. 
2) Medición mediante indicadores de la satisfacción de clientes, empleados, etc.

3) Comportamiento de la alta dirección

4) Gestión y mejora de los procesos

5) Gestión del personal, realizando cursos de formación, gestión y desarrollo.

6) Los mecanismos de mejora utilizados fueron:

- Equipos de proceso

- Equipos de unidad

- Formación de técnicas y herramientas de calidad

Para la mejora del proceso económico financiero de dicha empresa se utilizó la metodología más común en reingeniería:

1) Convergencia: Acordad funcionalidad del nuevo sistema

2) Construcción del nuevo sistema

3) Implantación del nuevo sistema

4) Post arranque o validación del nuevo sistema corrigiendo los fallos.

En conclusión la principal diferencia entre el BPR y el TQM en el caso anteriormente citado consiste en que la metodología utilizada para realizar un BPR está basada en un cambio radical de los procesos productivos de una empresa para obtener unos resultados mientras que el TQM está basado en un cambio incremental para lograr la mejora de la calidad total a partir de los procesos existentes.

La medición de resultados es muy compleja de medir en el ámbito del BPR, dado que existe gran número de cambios en reingeniería mientras que en el TQM es posible realizar una medición de los resultados mediante el modelo EFQM que permite realizar un diagnóstico interno de calidad.

La siguiente metodología estudiada está centrada en el artículo de Rodrigo Ospina duque en la cual, relaciona la reingeniería según Hammer y Champy (1993-1995) con la rápida reingeniería de Raymond, Manganelli y Klein (1995). La metodología para la reingeniería en dicho estudio está basada en las pautas sobre reingeniería de procesos de Mauricio Lefcovich (2006).

Según James Champy, cualquier cambio importante debe comenzar por describir el destino del viaje, es decir, hay que definir unos objetivos para conocer cuál es la meta a alcanzar.

También cita que la ruta a seguir por la organización consiste en realizar un enfoque hacia los procesos, empezando desde 0 en lugar de tratar rediseñar el proceso existente (rediseño radical). 
Según Lefcovich, las crisis en algunas organizaciones, dan lugar a el planteamiento de algunos empresarios a poner en marcha un proyecto de cambio o mejora en la empresa, basado en cuales son los motivos por los que debe hacerse el cambio y cuál es el alcance de dichos cambios, y por último cuál va a ser la implicación de la dirección en la gestión del cambio. Lefcovich define la reingeniería de procesos como $\ll$ Una recreación y reconfiguración de las actividades y procesos de la empresa, lo cual implica volver a crear y configurar los sistemas de la compañía〉>.

Hammer fue quien definió el proceso de Reingeniería como una respuesta a los desafíos de la internacionalización, mientras que Champy definía la reingeniería de procesos como una respuesta a la optimización de procesos.

El rediseño de procesos según Hammer y Champy plantea el estudiar y reestructurar completamente las organizaciones por parte de la gerencia para el cumplimiento de la misión de la empresa. La metodología que plantean consiste en el borrón y cuenta nueva, por lo que consiste en abandonar lo actualmente existente y partir de lo que los consumidores y clientes desean.

Todos los autores citados anteriormente coinciden en la metodología utilizada para la reingeniería de procesos, la cual está basada en las fases reingeniería propuestas por Manganelli y Klein:

1) Preparación del cambio: Consiste en mentalizar al personal que compone la organización de la necesidad de cambio para la consecución de los objetivos fijados por la empresa.

2) Planeación del cambio: Esta fase está basada en el estudio de las diferentes áreas de la empresa con el objetivo de identificar aquellos procesos y áreas de la empresa en las cuales existe una necesidad urgente de cambio.

3) Diseño del cambio: Fase en la cual se realiza el rediseño de los procesos productivos que necesitan mejora urgente así como implantación de las modificaciones.

4) Evaluación del cambio: consiste en la validación del rediseño o mejora de los procesos durante un periodo de tiempo en el cual se pueden corregir errores en dichos rediseños o mejoras.

Dentro de dichas fases existen una serie de procedimientos a seguir, nos centraremos en los citados por Lefcovich (2006):

- Definir el proyecto: que alcance tiene el proyecto y cuáles son los objetivos o metas que plantea. Debe basarse en la prospectiva de mercados y productos/servicios que desea cubrir, teniendo en cuenta la diferenciación con la competencia.

- Análisis de la situación actual: Realización de un diagnóstico de la situación actual de la organización. Se debe evaluar: 
- La organización: Historia, tecnología, productos y servicios ofrecidos, recursos utilizados, estrategias y políticas, prospectiva, estructura, etc.

- Entorno: Conocer la tendencia de demanda de los productos ofrecidos por la institución, características de los clientes, competidores, proveedores, conocer cuáles son las necesidades del cliente tanto interno como externo.

- Flujo de procesos: Información documentada de procesos tanto administrativos como técnicos, respondiendo a porque se hacen las cosas y como se hacen.

- Paradigmas empresariales: estudiar supuestos conscientes e inconscientes de la empresa y cuestionar los supuestos que no son válidos.

- Diagnóstico: Realizar un diagnóstico posterior al análisis en el cual se deben reflejar cuales son las necesidades más urgentes de la institución, y limitaciones y debilidades para poder llevar una gestión eficiente.

- Diseño de la nueva organización: Se recoge información de las etapas anteriores, y se crea una organización que cubra necesidades y limitaciones de la organización actual. Debe reflejar:

- Flujos de procesos: Los procesos que necesitan rediseño ya sea por un mal funcionamiento o no se realizan de forma adecuada.

- Flujos de información: Documentos, archivos o sus nuevos flujos para que la información fluya con un adecuado sistema de información gerencial.

- Organización: Diseño de características generales de la nueva estructura organizacional, tales como cargos, funciones, mercadeo de servicios, gestión financiera, cargas de trabajo y cultura organizacional.

- Estrategias y políticas: Nuevo sistema de gestión que va a seguir la organización.

- Paradigmas empresariales: Nuevas creencias y formas de hacer las cosas.

- Plataforma tecnológica: Determinar características y configuraciones necesarias para el software y el hardware.

- Productos o servicios: Proporcionar el producto o servicio con las características fundamentales para satisfacer las necesidades del cliente. 
- Implementación: Puesta en marcha del nuevo diseño o proyecto de organización, esto constituye uno de los pasos más complejos en el proceso de reingeniería debido a que el personal puede pensar que dichos cambios o rediseños pueden implicar la reducción de plantilla, en este paso es necesario reducir la incertidumbre del personal para que exista una buena implicación del personal, cosa indispensable para un buen funcionamiento del nuevo diseño de la organización.

- Validación: Esta última fase corresponde a la evaluación del nuevo diseño de la organización, en la cual mediante una serie de indicadores se puede medir el correcto desarrollo de los procesos rediseñados así como la obtención de los objetivos establecidos para dichos procesos. En esta fase se intenta corregir aquellos errores o deficiencias del nuevo diseño de la organización para que los procesos funcionen de forma eficiente, de acuerdo con las metas establecidas.

\section{RESULTADOS}

Los resultados obtenidos en este estudio del uso las diferentes metodologías utilizadas para la reingeniería de procesos nos muestra que cada uno de los autores utiliza metodologías muy semejantes a las de otros autores o son simplemente una evolución de las primeras metodologías de reingeniería de procesos creadas por Hammer y Champy, en las cuales se han incluido diferentes pasos a seguir para poder realizar un buen rediseño de los procesos productivos de forma que se optimicen dichos procesos.

\section{CONCLUSIONES}

Como conclusión del estudio podemos recalcar que la reingeniería de procesos (Business Process Reengineering) es una herramienta muy útil la cual nos permite conocer la necesidad de cambio en algunos procesos productivos de una empresa, estudiarlos y plantear diferentes metodologías que permitirán mejorar rendimiento de los procesos con la implantación de mejoras y con su posterior validación.

La reingeniería de procesos independientemente de la metodología utilizada persigue el mismo objetivo de alcanzar la meta deseada con respecto a la optimización de los procesos, consiguiendo siempre una implicación total del personal en los cambios propuestos para la mejora de la organización. 


\section{REFERENCIAS BIBLIOGRÁFICAS}

- Ospina Duque, Rodrigo. La reingeniería de procesos: una herramienta gerencial para la innovación y mejora de la calidad en las organizaciones.

- Escobar Pérez, Bernabé; María González González, José. Reingeniería de procesos de negocio: Análisis y discusión de factores críticos a través de un estudio de caso. Revista Europea de Dirección y Economía de la Empresa, vol. 16, núm. 3 (2007), pp. 93-114. ISSN 1019-6838.

- Escobar Pérez, Bernabé; María González González, José. Reingeniería de procesos y calidad total en la práctica empresarial: estudio de un caso. Revista de Contabilidad y Dirección Vol. 3, año 2006, pp 227-250

- Rafoso Pomar, Sandraliz; Artiles Bisbal, Sara. Reingeniería de procesos: conceptos, enfoques y nuevas aplicaciones. Ciencias de la Información Vol. 42, No.3, septiembre - diciembre, pp. $29 \quad-\quad 37,2011$ 